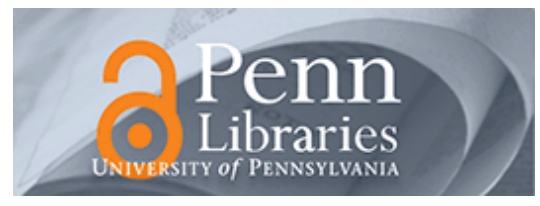

University of Pennsylvania

ScholarlyCommons

$10-1990$

\title{
Capillary Behavior of Binary Liquid Mixtures Near Criticality: Rise and Kinetics
}

Douglas J. Durian

University of Pennsylvania, djdurian@physics.upenn.edu

Kumudini Abeysuriya

Cornell University

Susan K. Watson

Cornell University

Carl Franck

Cornell University

Follow this and additional works at: https://repository.upenn.edu/physics_papers

Part of the Physics Commons

\section{Recommended Citation}

Durian, D. J., Abeysuriya, K., Watson, S. K., \& Franck, C. (1990). Capillary Behavior of Binary Liquid Mixtures Near Criticality: Rise and Kinetics. Physical Review A, 42 (8), 4724-4735. http://dx.doi.org/10.1103/

PhysRevA.42.4724

At the time of publication, author Douglas J. Durian was affiliated with Cornell University. Currently, he is a faculty member at the Physics Department at the University of Pennsylvania.

This paper is posted at ScholarlyCommons. https://repository.upenn.edu/physics_papers/636

For more information, please contact repository@pobox.upenn.edu. 


\title{
Capillary Behavior of Binary Liquid Mixtures Near Criticality: Rise and Kinetics
}

\author{
Abstract \\ In three different phase-separated binary liquid mixtures we have observed stationary capillary rises in \\ which the meniscus curvature is inconsistent with the sign of the rise. This "inverted-meniscus" \\ configuration occurs within approximately $50 \mathrm{mK}$ of the mixture's critical temperature and shows no sign \\ of decay after much longer than the characteristic time for relaxation. We also report experiments \\ showing that perturbation of the wetting layer inside the capillary tube can dramatically affect the \\ capillary rise. This motivates three scenarios in which the behavior of the wetting layer foils an \\ equilibrium capillary rise measurement of the contact angle and produces an inverted meniscus. \\ Disciplines \\ Physical Sciences and Mathematics | Physics

\section{Comments} \\ At the time of publication, author Douglas J. Durian was affiliated with Cornell University. Currently, he is a \\ faculty member at the Physics Department at the University of Pennsylvania.
}




\title{
Capillary behavior of binary liquid mixtures near criticality: Rise and kinetics
}

\author{
Douglas J. Durian \\ Laboratory of Atomic and Solid State Physics and Materials Science Center, Cornell University, \\ Ithaca, New York 14853-2501 \\ and Exxon Research and Engineering Company, Clinton Township, Route 22 East, Annandale, New Jersey 08801
}

Kumudini Abeysuriya, ${ }^{*}$ Susan K. Watson, and Carl Franck

Laboratory of Atomic and Solid State Physics and Materials Science Center, Cornell University, Ithaca, New York 14853-2501

(Received 14 May 1990)

\begin{abstract}
In three different phase-separated binary liquid mixtures we have observed stationary capillary rises in which the meniscus curvature is inconsistent with the sign of the rise. This "invertedmeniscus" configuration occurs within approximately $50 \mathrm{mK}$ of the mixture's critical temperature and shows no sign of decay after much longer than the characteristic time for relaxation. We also report experiments showing that perturbation of the wetting layer inside the capillary tube can dramatically affect the capillary rise. This motivates three scenarios in which the behavior of the wetting layer foils an equilibrium capillary rise measurement of the contact angle and produces an inverted meniscus.
\end{abstract}

\section{INTRODUCTION}

The phenomenon of capillary rise has been exploited by various researchers to investigate the wetting behavior of near-critical systems. ${ }^{1-6}$ These studies usually pit gravity against surface tension to infer the thermodynamic contact angle as a function of temperature, and thereby characterize the state of wetting. With refinements in both technique and sample preparation, capillary rise should continue to be a powerful tool in the search for, and study of, exotic wetting transitions, such as the partial drying ${ }^{6}$ or continuous wetting transitions (see, e.g., Refs. 7-10). However, a review of capillary-rise techniques for this purpose has not, to the best of our knowledge, appeared in the literature. Existing reviews ${ }^{11,12}$ emphasize capillary rise for the measurement of surface tension rather than of contact angle.

On one level, this paper illustrates the need for a systematic understanding of the technique and attempts to serve such a purpose. However, our initial motivation was to explore and understand a surprising, and highly reproducible, phenomenon dubbed the "inverted meniscus," which prevents the determination of contact angle from capillary rise. ${ }^{13}$ We begin by discussing in detail how the equilibrium capillary rise can be measured with confidence, and then demonstrate the procedures on three different binary liquid mixtures. We identify the time constant for capillary-rise relaxation and measure it as a function of temperature for two of these mixtures. Next, we present observations of the inverted meniscus showing how standard procedures can dramatically fail to give sensible contact angle results when within roughly $50 \mathrm{mK}$ of the critical temperature $T_{c}$. Possible explanations are proposed after we show that perturbation of the wetting layer which coats inside the capillary tube can significantly affect the capillary rise.

\section{EXPERIMENTAL MATERIALS AND PREPARATIONS}

The sample cells used for all measurements presented in this paper are similar to those described in Ref. 4, and used in Refs. 4-6. They consist of a 12-cm-long glass cylinder, inner diameter $0.9 \mathrm{~cm}$, whose bottom is fused shut and whose top can be sealed with a Teflon stopcock. The cells were filled with a critical composition binary liquid mixture, according to volume fraction, to a total liquid volume of approximately $7 \mathrm{ml}$. All reagents were obtained commercially, ${ }^{14}$ and were used without further purification. The critical temperature for each cell was measured visually to within $\pm 2 \mathrm{mK}$ to monitor sample purity and aging effects. Our thermostat was stable against temperature drifts to better than $1 \mathrm{mK} /$ day, and held temperature gradients (measured across $4 \mathrm{~cm}$ along the outside of the sample cell) to smaller than $1 \mathrm{mK} / \mathrm{cm}$.

All capillary tubes used in this study were cut from borosilicate glass micropipets. ${ }^{15}$ They had inner radii of $0.134,0.188$, or $0.300 \mathrm{~mm}$, and lengths typically between 4 and $6 \mathrm{~cm}$. The capillaries were held and manipulated inside the sample cell by a cylindrical Teflon holder which contained a Teflon-encapsulated magnetic stir bar (see Fig. 2 of Ref. 4). The cleaning procedures for all glass parts (capillaries and sample cell) were identical, or similar, to those described in Refs. 4 and 5. The basic procedure is to sonicate in changes of soap plus distilled water, acetone, methanol, ethylenediamine tetra-acetic acid (EDTA) solution, ${ }^{16}$ then $10 \%$ nitric acid, and to flush and sonicate with distilled water between each step. The cleaning procedures for all Teflon parts (capillary holder and stopcock) were identical, or similar, to those described in Refs. 4 and 5 ; i.e., sonication in soap plus distilled water followed by liberal rinsing, sonicating, and/or boiling with distilled water. We found that no systematic changes in the wetting 
behavior of clean, hydroxylated, capillaries were produced by variation of these procedures.

Carbon disulfide plus nitromethane. This mixture has a critical temperature of $T_{c} \simeq 63^{\circ} \mathrm{C}$ and a critical composition of $\phi_{c} \simeq 0.6014$ volume fraction of carbon disulfide. ${ }^{17}$ This system has $T_{c}$ drifts on the order of $+5 \mathrm{mK} / \mathrm{day}$. Equilibrium capillary-rise measurements have been reported as a function of $\left|T-T_{c}\right|$ and surface chemistry in Refs. 4-6. Clean, hydroxylated capillaries are completely wetted by the upper, nitromethane-rich phase and therefore normally exhibit a negative capillary rise (i.e., a capillary depression). From 18 to $0.5 \mathrm{~K}$ below $T_{c}$, our complete-wetting rise times radius data (from Ref. 6) obey the expected power law with amplitude $a_{0}^{2}=45 \pm 3$ $\mathrm{mm}^{2}$ [see Eq. (2) and Sec. III].

Cyclohexane plus acetic anhydride. This mixture has a critical temperature of $T_{c} \simeq 52^{\circ} \mathrm{C}$ and a critical composition of $\phi_{c} \simeq 0.613$ volume fraction of cyclohexane. ${ }^{17}$ Initial $T_{c}$ drifts were negative, but ceased after a few weeks; the value of $T_{c}$ was then constant to within $\pm 2 \mathrm{mK}$ over 4 months. Equilibrium capillary-rise measurements have been reported as a function of $\left|T-T_{c}\right|$ and surface chemistry in Ref. 5. Clean, hydroxylated capillaries are completely wetted by the lower, acetic anhydride-rich phase and therefore normally exhibit a positive capillary rise. From 40 to $1 \mathrm{~K}$ below $T_{c}$, our complete-wetting rise times radius data (from Ref. 5) obey the expected power law with amplitude $a_{0}^{2}=13.0 \pm 0.6 \mathrm{~mm}^{2}$ [see Eq. (2) and Sec. III].

2,6-lutidine plus water. This mixture has an inverted coexistence curve with a critical temperature of $T_{c} \simeq 33^{\circ} \mathrm{C}$ and a critical composition of $\phi_{c} \simeq 0.304$ volume fraction of lutidine. ${ }^{18}$ There was no noticeable $T_{c}$ drift: the value was constant to within $\pm 2 \mathrm{mK}$ over 9 months. Equilibrium rise measurements in borosilicate capillaries have been reported as a function of $T-T_{c}$ and added salt concentration in Refs. 1 and 3, respectively. In agreement with these references, we find that sufficiently clean borosilicate and quartz capillaries are both completely wetted by the lower, water-rich phase and therefore normally exhibit a positive capillary rise. ${ }^{19}$ From 8 to $0.1 \mathrm{~K}$ above $T_{c}$ (one order of magnitude closer than approached in Refs. 1 and 3), our new rise times radius data obey the expected power law with amplitude $a_{0}^{2}=120 \pm 10 \mathrm{~mm}^{2}$ [see Eq. (2) and Sec. III]. Going far above $T_{c}$, Refs. 1 and 3 both found that this system exhibits a transition to partial wetting by the water-rich phase.

\section{EQUILIBRIUM CAPILLARY RISE}

The equilibrium capillary rise $H_{\mathrm{eq}}$ in a tube of inner radius $r$ of a binary liquid mixture whose two coexisting phases have density difference $\Delta \rho$ and interfacial surface tension $\sigma$ is given by the capillary-rise formula ${ }^{11,12}$

$$
H_{\mathrm{eq}}=\frac{2 \sigma \cos \theta}{\Delta \rho g r} .
$$

Here $\theta$ is the contact angle and $g$ is gravitational ac- celeration. This formula represents the balance of hydrostatic pressure due to gravity and the Laplace pressure due to the curved interface. It is not valid if the rise is smaller than, or comparable to, the inner radius of the capillary. As discussed later, it also fails if the densities of the two phases inside the capillary are different from their respective values outside the capillary. References 4-6 demonstrate how Eq. (1) permits the contact angle of a partially wet capillary to be determined accurately by the ratio of its rise with that of a complete wet capillary.

Consider the case, as in all measurements reported here, of complete wetting by one of the phases, such that $|\cos \theta|=1$. The temperature dependence of the capillary rise is then a power law in reduced temperature $t=\left(T-T_{c}\right) / T_{c}$, which follows from the known ${ }^{11}$ power laws for the interfacial tension $\sigma=\sigma_{0}|t|^{\mu}$ and density difference $\Delta \rho=\rho_{0}|t|^{\beta}$ :

$$
H_{\mathrm{eq}} r=a_{0}^{2}|t|^{\mu-\beta}, \quad \mu-\beta=0.936 \pm 0.005 \text {. }
$$

The value of $\mu-\beta$ is taken from the well-established ${ }^{20}$ theoretical exponent estimates $\mu=1.264 \pm 0.002$ and $\beta=0.328 \pm 0.004$. Note that $a_{0}^{2}=2 \sigma_{0} / \rho_{0} g$ and is independent of the capillary radius.

This power-law prediction has been verified experimentally and values of $a_{0}^{2}$, noted earlier for the three binary liquid mixtures of this study, have been obtained as follows. After equilibrating the temperature of the sample cell, the liquid mixture was stirred thoroughly by raising and lowering the capillary tubes through the length of the cell. Next, sufficient time was allowed for the rain of bubbles in each of the phases to reach the bulk meniscus, leaving two clear coexisting phases of equilibrium composition. Agitation was often required to dislodge bubbles inside the capillaries, especially if they happened to be submerged in the nonwetting phase. Once all bubbles were gone and the two phases perfectly clear, the capillaries were slowly raised through the bulk meniscus. After the rise inside the capillary reached a stationary value, it was measured with a cathetometer ${ }^{21}$ of resolution $0.01 \mathrm{~mm}$. Note that these procedures ensure that the liquid phases inside the capillary had the same compositions as outside. Several measurements were then made by repositioning the capillaries with respect to the bulk meniscus and measuring the rise after reequilibration. Since the meniscus inside the capillary barely moves while the capillary is repositioned, the approach to the new equilibrium position can be chosen as advancing or receding ${ }^{22}$ by either raising or lowering the capillaries, depending on which phase wets the substrate. For the case of complete wetting far enough from $T_{c}$ that the rise is much greater than the radius, we found the same results for both advancing and receding directions.

Inspection of $H_{\mathrm{eq}} r$ versus $\left|T-T_{c}\right|$ data in the log-log plot of Fig. 1 reveals power-law behavior which could be fitted for exponent and amplitude $a_{0}^{2}$. Instead, the amplitude values quoted earlier were obtained by forcing the exponent to be $\mu-\beta=0.936$ and fitting only for $a_{0}^{2}$; these fits are included in Fig. 1. Note that this method of analysis is a more stringent test of the predicted power law (2) than if the exponent were also adjusted. It also 


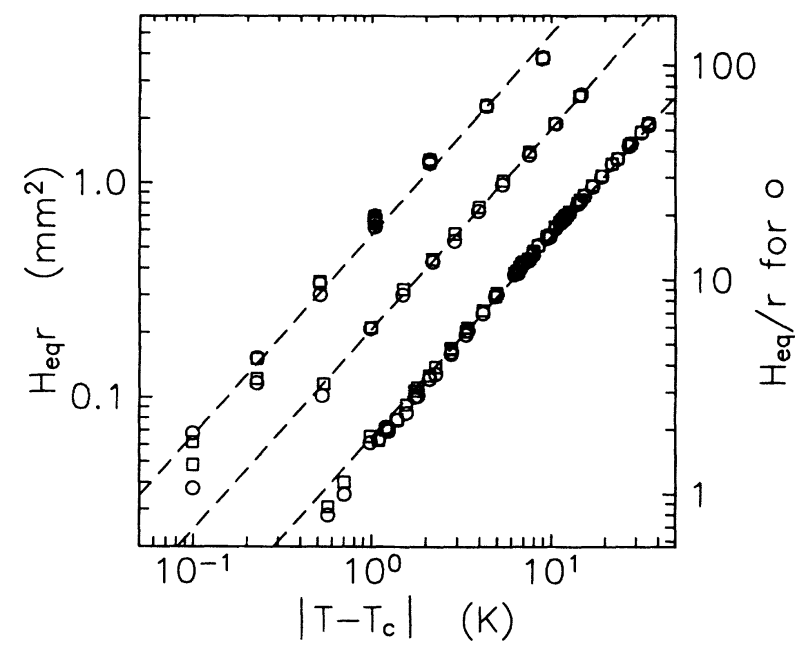

FIG. 1. Capillary-rise times radius vs temperature for three binary liquid mixtures: 2,6-lutidine plus water (left set), carbon disulfide plus nitromethane (middle set), and cyclohexane plus acetic anhydride (right set). The dashed lines are the fits to Eq. (2) for amplitude $a_{0}^{2}$, keeping the exponent fixed at $\mu-\beta=0.936$. Open (solid) symbols are for receding (advancing) meniscus directions; squares (circles) represent $0.134-(0.188-)$ mm-radii capillaries. The right axis shows capillary rise in units of radius for the larger capillary tube $r=0.188 \mathrm{~mm}$.

provides a more reliable value of the amplitude $a_{0}^{2}$. As described in Ref. 6, a correction due to the mislocation of the bulk meniscus was first performed on our 2,6-lutidine plus water and carbon disulfide plus nitromethane capillary-rise data.

\section{KINETICS OF CAPILLARY RISE}

This section concerns the kinetics of capillary-rise relaxation after the repositioning procedure described above. The purpose is to demonstrate our certainty that sufficient time had passed before capillary-rise values were measured. To good approximation, the relaxation can be described by the Washburn equation as a balance of hydrostatic and interfacial curvature pressures with viscous forces from flow inside the capillary. ${ }^{23,24}$ Neglecting inertial and entrance effects ${ }^{24}$ and assuming that the viscosities of the liquid phases are well matched (a good approximation sufficiently close to $T_{c}$ ), we find that the predicted relaxation is exponential:

$$
H(\tau)-H_{\text {eq }} \propto \exp \left(-\tau / \tau_{0}\right), \quad \tau_{0}=\frac{8 \eta L}{\Delta \rho g r^{2}} .
$$

Here $\tau$ is time, $\tau_{0}$ is the relaxation time constant, $\eta$ is the dynamic viscosity, and $L$ is the length of the capillary. Therefore $\tau_{0} r^{2} / L$ is independent of $r$ and $L$, and should have the power-law temperature dependence $|t|^{-\beta}$, assuming that viscosity is independent of temperature. Note that this prediction is the same for both advancing and receding directions.

We have examined Eq. (3) experimentally for two binary liquid systems by measuring the capillary rise

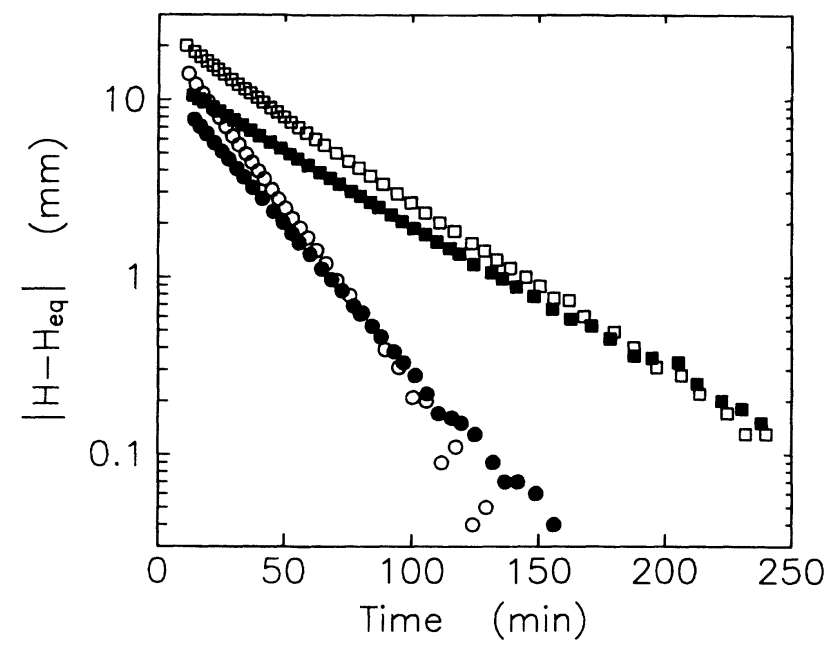

FIG. 2. Capillary rise vs time after repositioning for 2,6lutidine plus water at $T_{c}+20 \mathrm{mK}$. The relaxations are exponential, as predicted by Eq. (3). Open (solid) symbols are for receding (advancing) meniscus directions; squares (circles) represent the $0.134-(0.188-) \mathrm{mm}$-inner-radius capillary. Contrary to expectation, here the receding menisci relax faster than advancing ones.

versus time after repositioning. For carbon disulfide plus nitromethane we observed the relaxation in a $0.134-\mathrm{mm}$ radius capillary; for 2,6-lutidine plus water we observed the relaxation in both $0.134-$ and 0.188 -mm-radii capillaries, an example of which is shown in Fig. 2. The lengths were always $L=6 \mathrm{~cm}$. In every observation the relaxation was exponential over the entire range of resolution: tens to hundredths of millimeters. Time constants were extracted from semilogarithmic plots and are displayed in Fig. 3. Note that in these experiments the

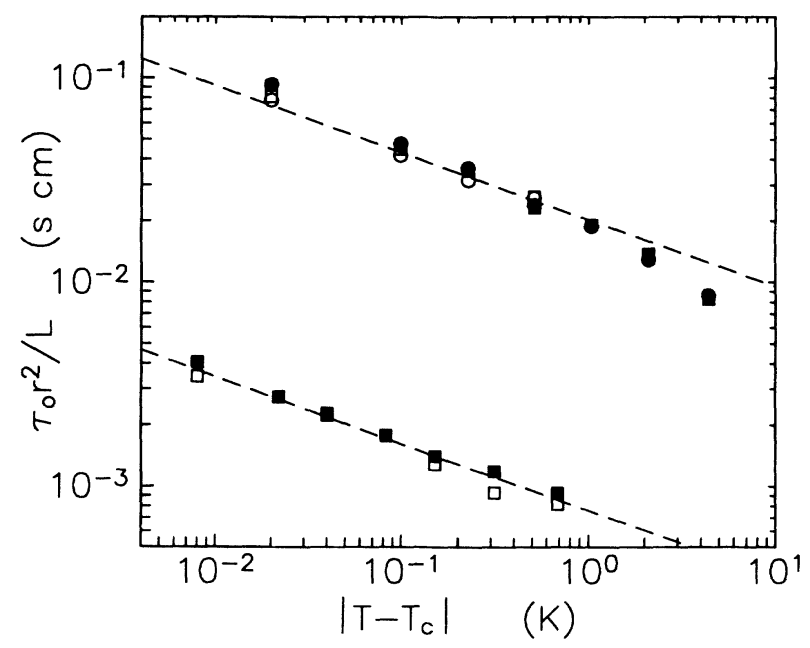

FIG. 3. Capillary-rise relaxation time vs temperature for 2,6-lutidine plus water (upper set) and carbon disulfide plus nitromethane (lower set). These data are scaled with the radius $r$ and length $L$ of the capillary according to Eq. (3), and show the expected power-law temperature dependence (dashed line). Open (solid) symbols are for receding (advancing) meniscus directions; squares (circles) represent 0.134- (0.188-) mm-innerradii capillaries. 
fluid motion is very slow, as characterized by the Reynold's number $\mathcal{N}_{\mathrm{Re}}=\rho r V / \eta$, where $V$ is the average flow speed and $\rho$ is the mass density. The smallest time constant observed was $\tau_{0}=31 \mathrm{~s}$ for carbon disulfide plus nitromethane at $684 \mathrm{mK}$ below $T_{c}$ in a $0.134-\mathrm{mm}$-innerradius capillary. From this case, we estimate that $\mathcal{N}_{\mathrm{Re}} \simeq 0.1$ was the largest Reynold's number attained in our experiments.

For the carbon disulfide plus nitromethane mixture, a very good fit to all the relaxation data is given by $\tau_{0} r^{2} / L=(1.13 \pm 0.08 \mathrm{~s} \mathrm{~cm}) \times 10^{-4}|t|^{-\beta}$, as shown in Fig. 3. At some temperatures, the relaxation in the receding meniscus direction seems to be faster than in the advancing direction; however, the difference is not much larger than our estimated uncertainty.

For the case of 2,6-lutidine plus water, the time constants for different radii tubes collapse according to Eq. (3), but we could not obtain a fit to the appropriate power law of comparable quality to the previous mixture. Again fixing the value of $\beta$, we obtain $\tau_{0} r^{2} / L=(31 \pm 5$ $\mathrm{s} \mathrm{cm}) \times 10^{-4}|t|^{-\beta}$, as shown in Fig. 3. The failure to obtain an excellent fit over the entire temperature range may be due to the decrease of viscosity with increasing temperatures (consistent with the systematic deviation seen at large $T-T_{c}$ in Fig. 3). Just as for carbon disulfide plus nitromethane, at most temperatures we find no significant difference between advancing and receding directions. However, contrary to the prediction of the Washburn equation, at $T-T_{c}=20 \mathrm{mK}$ we find a smaller time constant for the receding case to well within our uncertainty. This can be readily seen in Fig. 2. To repeat, near $T_{c}$ a receding meniscus relaxes faster than an advancing meniscus. The wetting layer structure near the moving meniscus might be reponsible.

\section{INVERTED MENISCUS}

The procedures developed and followed above for measuring the equilibrium rise of a binary liquid mixture inside a capillary tube can be used with confidence sufficiently far from $T_{c}$. Nevertheless, in this section we present a series of observations on three different mixtures near $T_{c}$ in which these same procedures dramatically fail. We consistently observed an "inverted-meniscus" phenomenon in which the sign of the capillary rise contradicted the meniscus curvature. As $T_{c}$ was approached, the equilibrium capillary rise at first decreased in accord with Eq. (2). Then sufficiently close to $T_{c}$, we found that the capillary rise could change sign even though the curvature of the meniscus did not. This configuration is a blatent violation of the usual equilibrium achieved by a balance of hydrostatic and capillary pressures, represented by the capillary-rise formula (1), yet it persists much longer than the characteristic time for relaxation. We call such an observation "stationary," but cannot conclude that the configuration is truly steady state. For clarity we depict normal- and invertedmeniscus configurations in Fig. 4. In Fig. 5 we display a photograph of an inverted meniscus at $T_{c}-35 \mathrm{mK}$ inside a $0.188-\mathrm{mm}$-inner-radius capillary tube containing carbon

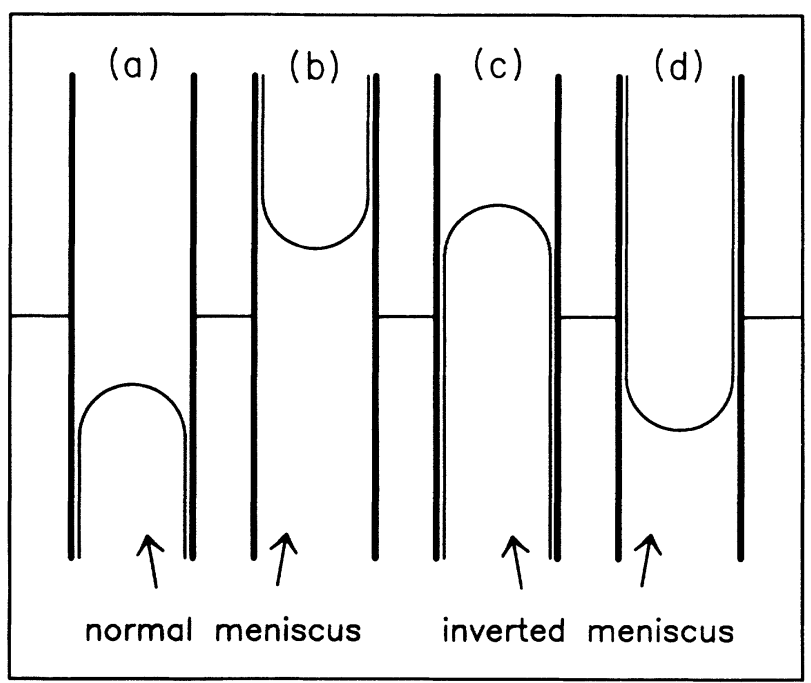

FIG. 4. Schematic of (a) and (b) normal and (c) and (d) inverted meniscus configurations. (a) and (c) for carbon disulfide plus nitromethane, the upper, nitromethane-rich, phase wets glass. (b) and (d) for 2,6-lutidine plus water, the lower, waterrich, phase wets glass. (b) and (d) for cyclohexane plus acetic anhydride, the lower, acetic anhydride-rich, phase wets glass. Note that the wetting layer thickness has been greatly exaggerated for clarity.

disulfide plus nitromethane: the capillary rise is positive even though the curvature clearly indicates wetting by the upper case. This photograph was taken long after a stationary value of the rise had been reached. Note that the extreme flatness of the bulk meniscus simplifies observation of the inverted meniscus.

Our experience was that very near $T_{c}$ an inverted meniscus usually appeared. Occasionally, however, the inverted meniscus was not found, either because it was obscured by the bulk meniscus or because it was too flat to ascertain its curvature. We have never observed its curvature to change sign with respect to its value further from $T_{c}$. In Fig. 6 we present both a typical observation and nonobservation of an inverted meniscus at $20 \mathrm{mK}$ into the two phase region of a 2,6-lutidine plus water mixture inside a $0.188-\mathrm{mm}$-inner-radius capillary. As also seen further away from $T_{c}$, the meniscus curvature indicated wetting by the lower phase. At time zero the capillary tube was raised through the bulk meniscus and the height of the meniscus inside the capillary was monitored as it receded down toward, and then through, the bulk meniscus. A stationary inverted meniscus was attained by $100 \mathrm{~min}$ and persisted unchanged for the duration of the observation. Next, time was reset to zero and the capillary was repositioned a few centimeters lower. The meniscus inside the capillary was then monitored as it advanced upward and disappeared into the bulk meniscus. This represents a null observation of the inverted meniscus for the advancing meniscus direction.

Figure 7 presents observations of the inverted meniscus in a third mixture: cyclohexane plus acetic anhydride. 


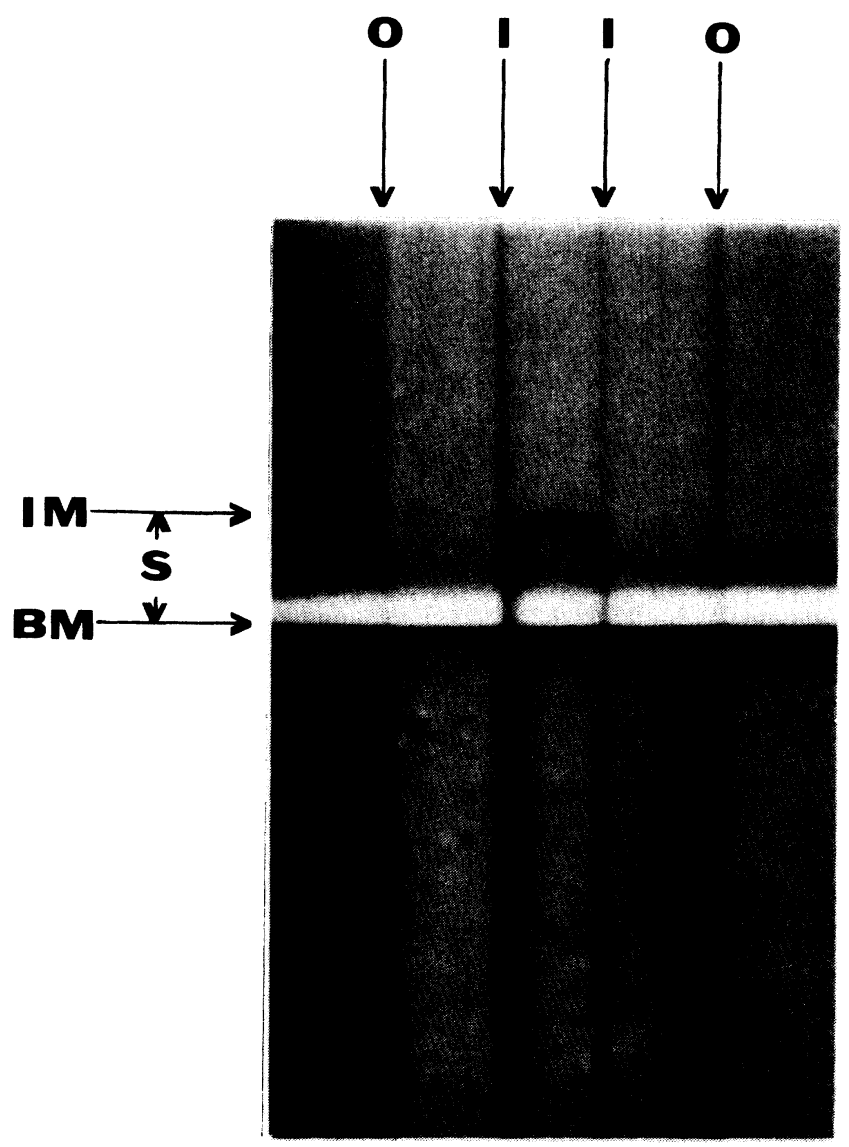

FIG. 5. Photograph of the inverted meniscus in a $0.188-\mathrm{mm}$ inner-radius capillary at $35 \mathrm{mK}$ below the critical temperature of a carbon disulfide plus nitromethane mixture. $\mathbf{O}$ (I) indicates the outer (inner) wall of the capillary tube; BM and IM indicate the respective bulk and inverted meniscus positions; and $s$ is the capillary rise. Note that $s$ is positive even though the meniscus curvature is clearly visible and implies wetting by the upper phase.

Here we tracked the capillary rise in four capillaries versus time for $114 \mathrm{~h}$. The meniscus curvatures in this mixture indicated wetting by the lower phase, so the negative capillary rise implies an inverted meniscus. This figure again shows that the inverted meniscus can exist in different mixtures, and that it truly persists for times much greater than the characteristic time for capillaryrise relaxation (equal to aproximately $10 \mathrm{~min}$ here). For the carbon disulfide plus nitromethane mixture, our longest observation of an inverted meniscus was $21 \mathrm{~h}$ for receding initial conditions, and $5 \mathrm{~h}$ for advancing.

It is also interesting to note in Fig. 7 that the scatter in the data is strongly correlated for all capillaries. This suggests that some external condition, such as mechanical vibrations or temperature fluctuations, can influence the capillary rise. The latter possibility will be examined in later sections.

In Fig. 8 we return to the carbon disulfide plus nitromethane mixture and look for a systematic dependence of the inverted meniscus upon $\left|T-T_{c}\right|$ and the ra-

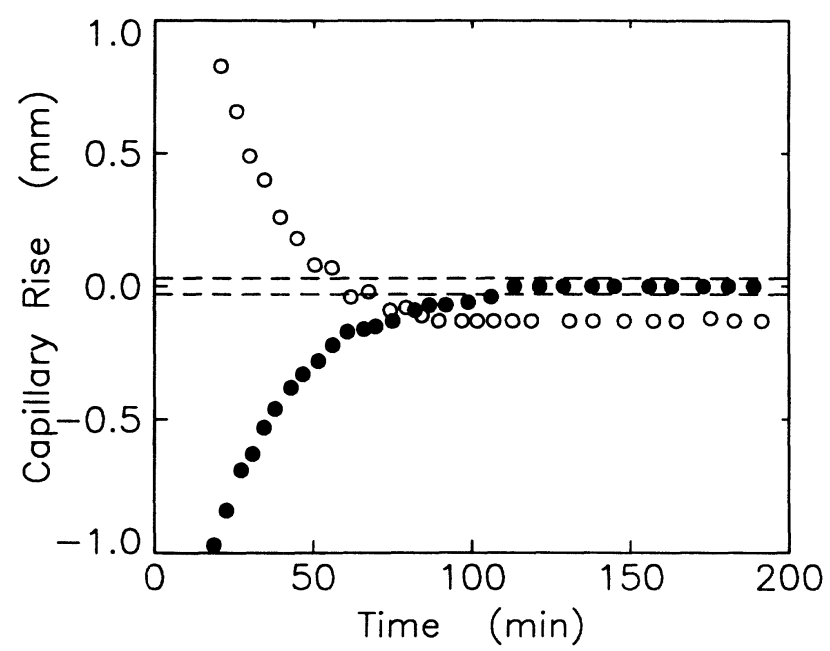

FIG. 6. Capillary rise vs time after repositioning for 2,6lutidine plus water at $T_{c}+20 \mathrm{mK}$ inside a 0.188 -mm-innerradius capillary (these data were shown earlier in Fig. 2). Note that in the receding case (open circles) the rise reached a stationary negative value even though the observed curvature indicated wetting by the lower phase: this is an inverted meniscus. For the advancing meniscus (solid circles) the rise became obscured in the bulk meniscus: this is a normal meniscus. The dashed lines indicate the range over which curvature of the bulk meniscus may interfere with observation.

dius. There is large scatter in the stationary values of the capillary rise, but in most observations it was either positive, and hence, inverted, or else hidden by the bulk meniscus. In a few exceptional cases we could not ascertain the curvature of the meniscus. The rise of the inverted meniscus seemed to increase for small $\left|T-T_{c}\right|$, but we

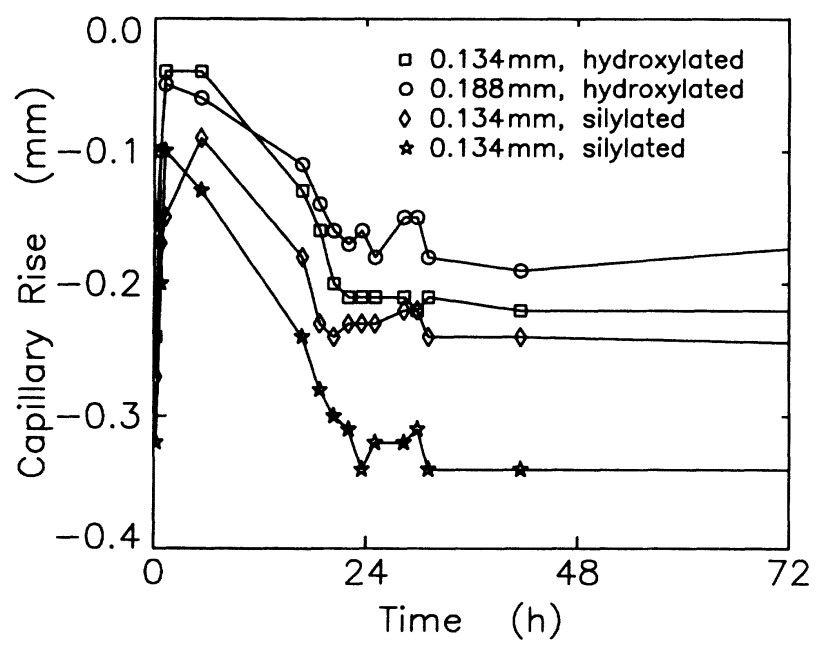

FIG. 7. Inverted meniscus height vs time for cyclohexane plus acetic anhydride at $45 \mathrm{mK}$ below $T_{c}$; the final data points (not shown) were collected at $114 \mathrm{~h}$. Inner radii and surface chemistry are as specified. The silylated capillaries were reacted with the vapor of hexamethyldisilazane according to the procedures of Ref. 5. Note the strong correlation of fluctuations in these data. 


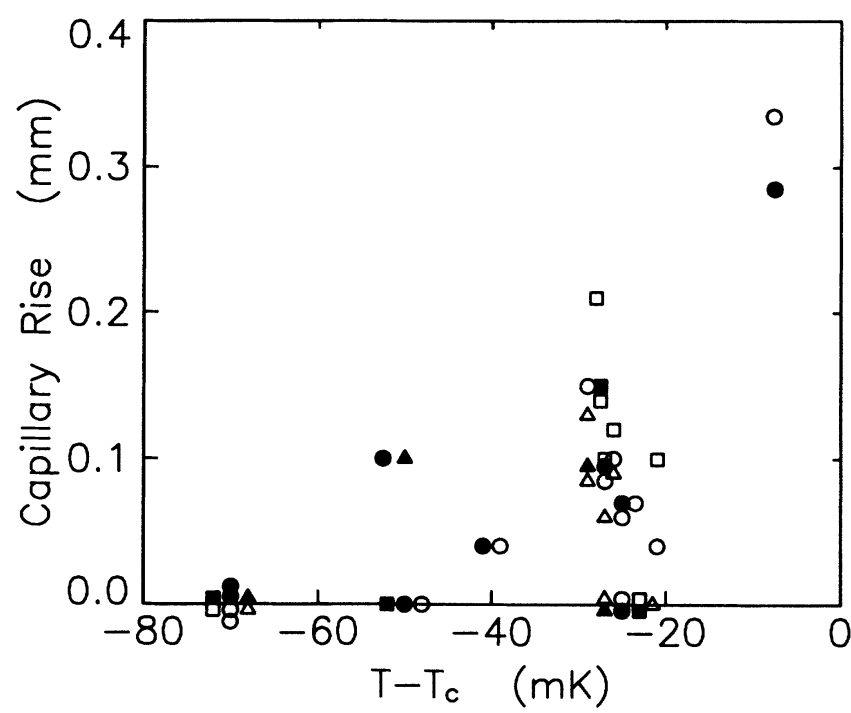

FIG. 8. Stationary capillary rise vs temperature in carbon disulfide plus nitromethane near the critical temperature $T_{c}$. In all cases that the meniscus curvature could be detected, it indicated wetting by the upper phase: therefore positive rises are observations of the inverted meniscus. Points located within $0.02 \mathrm{~mm}$ of zero correspond to no detectable rise, and were slightly displaced for clarity. Open (solid) symbols are for receding (advancing) meniscus directions; squares, circles, and triangles, respectively, represent $0.134-, 0.188-$, and $0.300-\mathrm{mm}$ inner-radii capillaries.

observed no particular trend versus capillary radius. Note that these observations were made for both advancing and receding initial conditions.

Continuing with carbon disulfide plus nitromethane, we look for a systematic dependence of the inverted meniscus upon the position of the capillary tube with respect to the bulk meniscus in Fig. 9. This was performed at 20 $\mathrm{mK}$ below $T_{c}$ for two different radii capillary tubes. We denote the capillary tube position by $l_{2}$, the length by which it extends into the nonwetting phase. We find much scatter in the data, but Fig. 9 suggests an increase of the effect with $l_{2}$. These data cannot, however, unambiguously rule out the possibility of no dependence upon $l_{2}$.

In Fig. 10 we repeat the previous experiment for the mixture cyclohexane plus acetic anhydride at $45 \mathrm{mK}$ below $T_{c}$. Now we clearly observe a dependence of the stationary rise on the position of the capillaries. As $l_{2}$ is increased, a normal meniscus is turned into an inverted meniscus, which then continues to increase. We find that the meniscus position seems to vary linearly with $l_{2}$.

To conclude this section, we have consistently observed the inverted meniscus close to $T_{c}$ in all three binary mixtures examined. This configuration is stationary in that it persists without any sign of decay for much longer than the time ordinarily expected for capillary-rise relaxation. The size of the effect seems to increase with $l_{2}$, the length by which the capillary extends into the nonwetting phase, and with decreasing $\left|T-T_{c}\right|$. We can

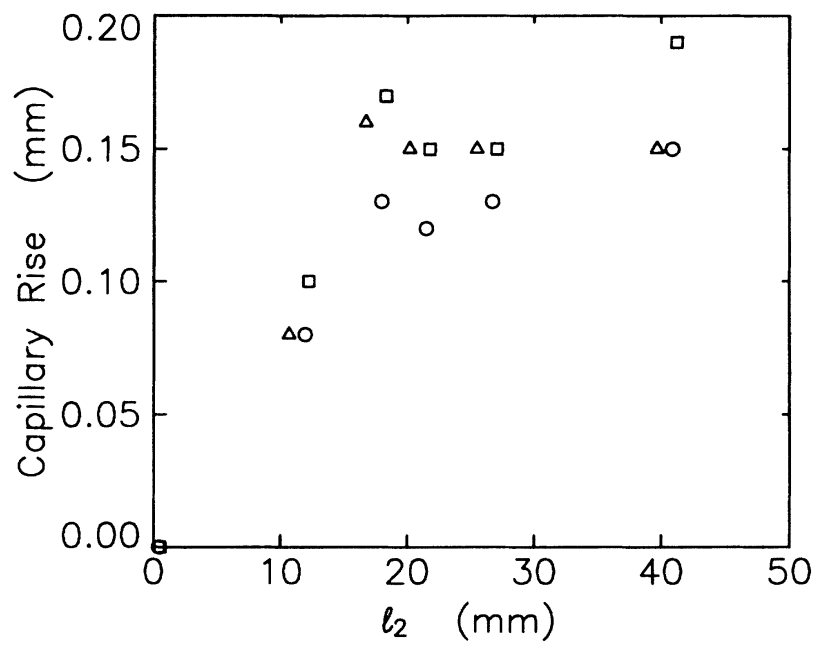

FIG. 9. Stationary capillary rise vs capillary tube position in carbon disulfide plus nitromethane at $20 \mathrm{mK}$ below $T_{c}$ for initially advancing meniscus direction. Same symbol convention as in Fig. 8. In spite of the large scatter, it is possible to conclude that the rise increases with $l_{2}$, the length by which the capillary extends into the lower, nonwetting, phase.

distinguish no systematic dependence on the capillary radius.

\section{TEMPERATURE PERTURBATION EXPERIMENTS}

In Ref. 25 it was reported that a temperature perturbation as small as a few millikelvin at $15 \mathrm{~K}$ below $T_{c}$ (i.e.,

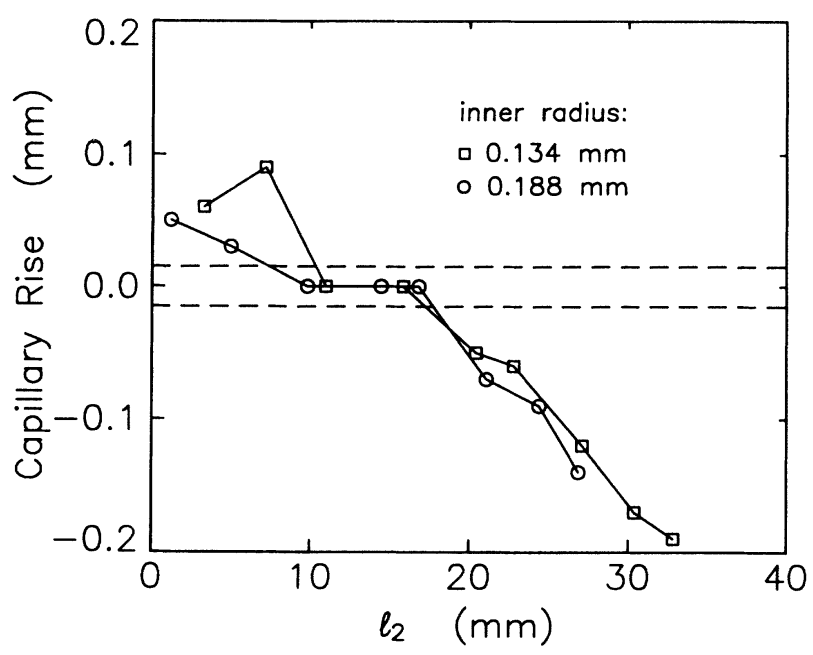

FIG. 10. Stationary capillary rise vs capillary tube position for cyclohexane plus acetic anhydride at $45 \mathrm{mK}$ below $T_{c}$ for initially receding meniscus direction. Since the meniscus curvature indicated wetting by the lower phase, this figure shows that the menisci became inverted as the extension of the capillary into the nonwetting phase $l_{2}$ increased. The total length of the capillary tubes was $50 \mathrm{~mm}$. The dashed lines indicate the range over which curvature of the bulk meniscus may interfere with observation. Squares (circles) represent $0.134-(0.188-)$ mm-radii tubes. 
$0.01 \%$ ) can cause the wetting layer thickness to change temporarily by more than $10 \%$ before relaxing back to the same equilibrium value. It was observed that a temperature "quench" (further into the two-phase region) causes the wetting layer to thicken, and that a "burn" (toward $T_{c}$ ) causes the layer to thin down. Equation (2) of Ref. 25 predicts that the change in wetting layer thickness $\delta$ resulting from a small temperature change $\Delta T$ will be

$$
\left.\delta \simeq \frac{\beta \Delta T}{2\left(T-T_{c}\right)}\left|\frac{d_{0}}{\sigma} \frac{\partial^{2} V}{\partial z^{2}}\right|_{z_{\mathrm{eq}}}\right)^{-1},
$$

where $\beta \simeq 0.328, d_{0}$ equals approximately one-sixth of the correlation length, $\sigma$ is the interfacial surface tension, $V(z)$ is the effective interface potential, and $z_{\text {eq }}$ is the equilibrium thickness of the wetting layer. This implies that the sensitivity of the wetting layer to temperature perturbations should become even greater near $T_{c}$. Therefore it is prudent to consider how close one can approach the critical temperature before the small temperature variations due to the surrounding bath begin to influence the measurement. In this spirit, we now investigate the effect of temperture perturbations on the capillary rise.

In Fig. 11 we show the response of the capillary rise of a phase-separated carbon disulfide plus nitromethane mixture, initially equilibrated at $T_{c}-165 \mathrm{mK}$, to a 50 $\mathrm{mK}$ burn. After liquid equilibration, the capillaries were positioned through the bulk meniscus. The meniscus in each tube then advanced to its equilibrium position in less than $10 \mathrm{~min}$. At about $25 \mathrm{~min}$ the temperature burn was started; 5 min later the temperature restabilized 50

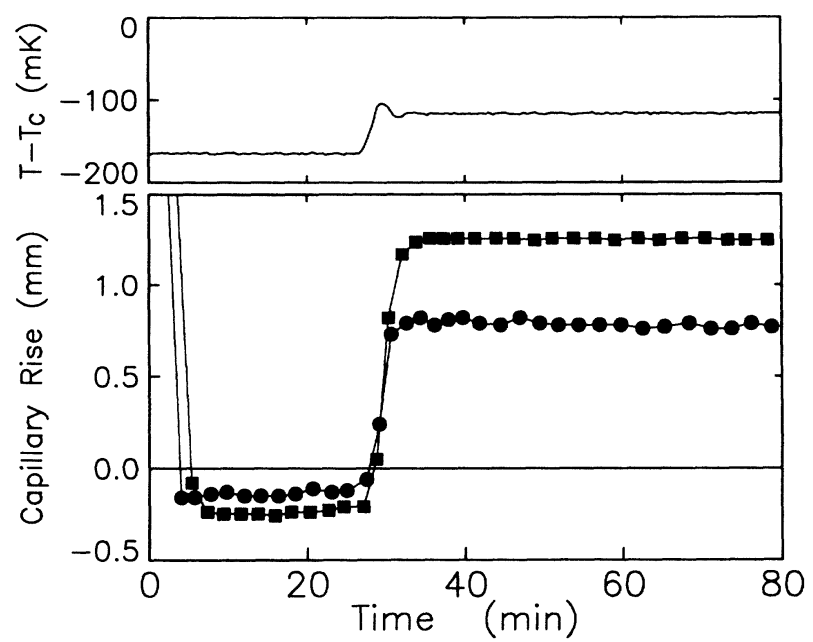

FIG. 11. Effect of a temperature jump on the capillary rise of carbon disulfide plus nitromethane. At time zero the tubes were moved into position and their rises subsequently recorded as a function of time. After about $25 \mathrm{~min}$ the rises had long reached a stationary value and a large temperature jump was applied, producing the dramatic results shown. In these observations the meniscus curvatures always indicated wetting by the upper phase, even after the rise changed sign. Squares (circles) represent $0.134-(0.188-)$ mm-radii tubes.

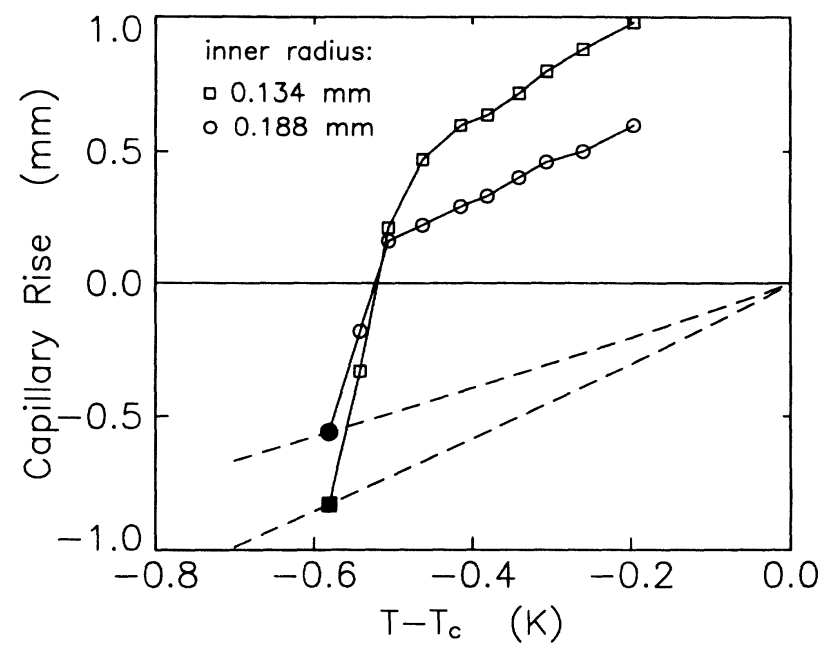

FIG. 12. Effect of successive temperature jumps on the capillary rise of carbon disulfide plus nitromethane. First the liquids were equilbrated at $0.581 \mathrm{~K}$ below $T_{c}$ and the equilibrium rise was measured (solid symbols). Next the temperature was increased slightly; after several minutes the capillary rise stopped changing and was recorded. Continuing in this manner successive temperature jumps were performed, with the results collected here for 0.134- and 0.188-mm-radii capillaries ( $\square$ and $\bigcirc$, respectively). The dashed curves show the temperature dependence of the expected equilibrium capillary rise: $\left|T-T_{c}\right|^{\mu-\beta}$.

$\mathrm{mK}$ closer to $T_{c}$. The response of the rise in two capillaries to this perturbation is dramatic, and larger for the smaller radius capillary. The meniscus in each capillary receded toward, and then through, the bulk meniscus all the while never changing its curvature. After several minutes the rises stabilized far above the bulk meniscus, as shown, convincingly mimicking an inverted meniscus. Presumably if we had then waited a diffusion time for the entire sample, the system would have reequilibrated according to Eq. (1) such that the rise in each tube would again be negative, but closer to the bulk meniscus. We contend that the effect demonstrated in Fig. 11 is due to the sensitivity of the wetting layer inside the capillary tube to temperature perturbations. This will be discussed after presentation of further experiments.

In Fig. 12 we show the effect of successive temperature perturbations on the capillary rise of carbon disulfide plus nitromethane. First the equilibrium rise was measured at $581 \mathrm{mK}$ below $T_{c}$ for two different radii capillaries. Next a small temperature burn was applied and the restabilized value of the capillary rise was recorded. Continuing in this manner further temperature jumps were applied and the restabilized rises were found as shown. As in Fig. 11, note that the response is greater for the smaller radius capillary tube.

In Fig. 13 we compare the effect of successive temperature burns on the rise inside the 0.134 -mm-inner-radius capillary for two different positions with respect to the bulk meniscus. Similar to the inverted meniscus of Sec. $\mathrm{V}$, we observe that the effect of a temperature perturbation is greater roughly in proportion to $l_{2}$, how far the 


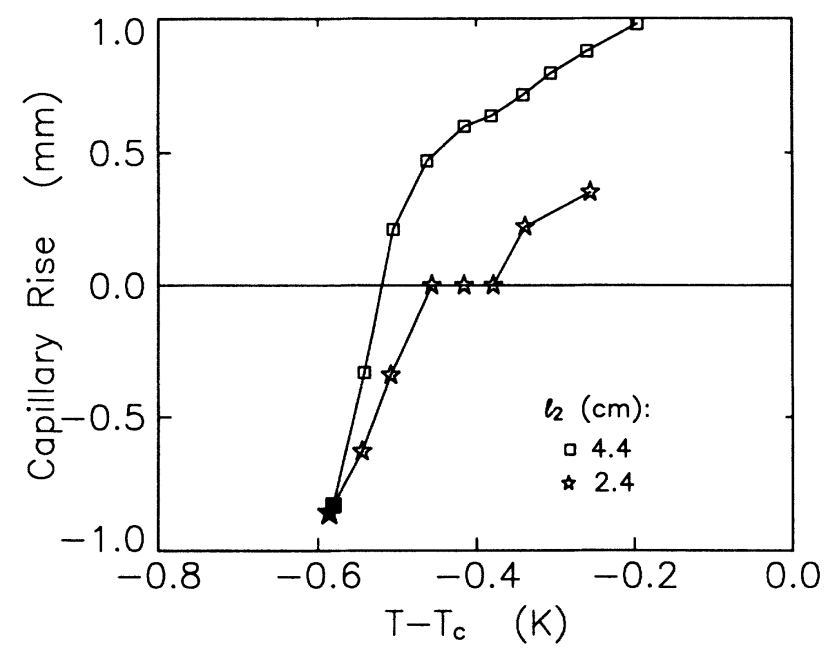

FIG. 13. Successive temperature jump experiments performed as in Fig. 12. Shown here are results for two runs in which the same capillary tube is positioned with respect to the bulk meniscus as indicated. Note that the response of the tube inserted further into the nonwetting phase (larger $l_{2}$ ) is somewhat less than twice as great as that of the other. The solid symbols indicate equilibrium capillary-rise measurements.

capillary extends into the nonwetting phase.

The final temperature perturbation experiment is shown in Fig. 14, again using the mixture of carbon disulfide plus nitromethane. There we equilibrated at

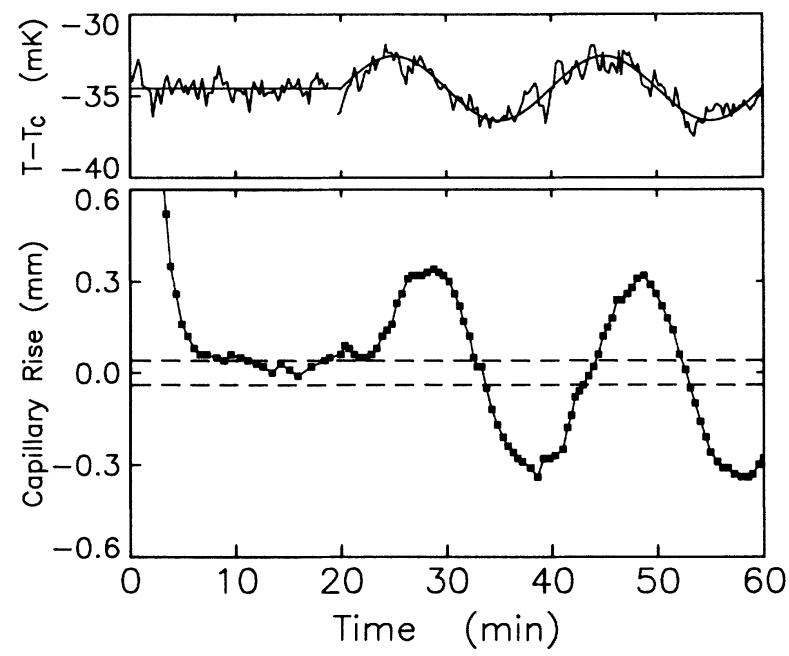

FIG. 14. Effect of a small temperature oscillation on the capillary rise of carbon disulfide plus nitromethane in a 0.134 mm-inner-radius capillary tube. At time zero the tube was moved into position and its rise subsequently recorded as a function of time. After $20 \mathrm{~min}$ the rise had long reached a steady value and a 2 -mK-temperature oscillation was applied (the smooth curve through the temperature data represents the controlling voltage). Throughout these observations the meniscus curvature always indicated wetting by the upper, nitromethane-rich, phase. The dashed lines indicate the range over which curvature of the bulk meniscus may interfere with observation.
$34.5 \mathrm{mK}$ below $T_{c}$ and positioned the capillary through the bulk meniscus. The meniscus inside the capillary advanced toward the bulk meniscus and stopped moving within $10 \mathrm{~min}$. It was sufficiently close that the bulk meniscus partially obscured the meniscus inside the capillary; but the rise seemed to stop at a positive value (and is therefore another observation of the inverted meniscus). At 20 min a sinusoidal temperature oscillation was applied with a 2-mK amplitude (slightly larger than the precision of our temperature control). The capillary-rise reponse is evidently large, being somewhat larger than typical inverted-meniscus heights. This demonstrates that in the region near $T_{c}$ where an inverted meniscus is observed, the sensitivity of capillary rise to temperature perturbations is great enough that fluctuations due to the surrounding water bath could have some effect. In particular, it suggests that imperfect temperature control should be considered in the search for an explanation of the inverted meniscus.

\section{GENERALIZED CAPILLARY-RISE FORMULA}

In the experiments of Sec. VI, the wetting layer coating the inside of the capillary tube must decrease in thickness following the temperature burn. ${ }^{25}$ The wetting layer material shed during this process will diffuse to the interior of the capillary, thereby slightly changing the average density of the liquid inside the tube. The resulting effect on the hydrostatic force balance may explain our temperature perturbation experiments.

Let $\rho_{C}$ and $\rho_{N}$ be the densities of the bulk carbon disulfide-rich and nitromethane-rich phases, respectively, and call the change in wetting layer thickness $\delta$ (negative for the decrease in thickness now being considered). Assuming that the shedded material goes to the interior of a capillary of radius $r$, the new average density of the carbon disulfide-rich phase inside the capillary will be $\rho_{C}^{\prime}<\rho_{C}$ such that

$$
\frac{\rho_{C}^{\prime}-\rho_{C}}{\Delta \rho} \simeq \frac{2 \delta}{r},
$$

where $\Delta \rho \equiv \rho_{C}-\rho_{N}$. The effect on the hydrostatic balance, which determines the capillary rise, can be found by balancing the pressure changes around the closed circuit shown in Fig. 15. Starting at the lower left corner of the circuit and proceeding clockwise, the sum of pressure drops must be zero:

$$
\rho_{C} g l_{2}+\rho_{N} g l_{1}-\rho_{N} g\left(l_{1}-H\right)-\frac{2 \sigma}{r}-\rho_{C}^{\prime} g\left(l_{2}+H\right)=0 .
$$

Here $g$ is gravitational acceleration, $l_{1}$ and $l_{2}$ are the vertical circuit lengths defined in Fig. 15, $H$ is the capillary rise, and $\sigma$ is the interfacial surface tension; the case of complete wetting by the upper phase is assumed. In keeping with our earlier definition, $l_{2}$ is the length by which the capillary extends into the nonwetting phase. Equation (6) can be solved for $H$, yielding a generalized capillary-rise formula: 


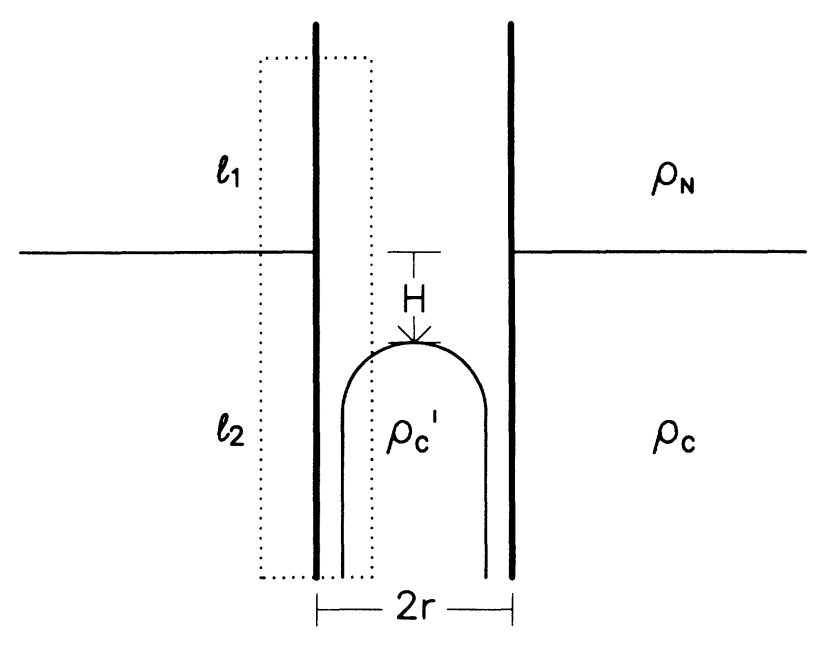

FIG. 15. Schematic of capillary rise $H$ inside a tube of radius $r . \rho_{N}$ and $\rho_{C}$ are the two bulk liquid densities, and $\rho_{C}^{\prime}$ is the liquid density of the lower phase inside the capillary tube. $l_{1}$ and $l_{2}$ indicate the size of the circuit in each phase as used to derive an expression for $H$; the circuit is taken such that $l_{2}$ is the length which the capillary tube extends into the nonwetting phase. $H$ is measured from the bulk meniscus and is taken to be negative for the capillary depression shown here. After a small temperature "burn" toward $T_{c}$, wetting layer material will be expelled to the interior of the capillary causing the density change $\rho_{C}^{\prime}-\rho_{C}<0$; this causes the meniscus to recede upward. Note that the wetting layer thickness has been greatly exaggerated for clarity.

$$
H=-\frac{\rho_{C}^{\prime}-\rho_{C}}{\Delta \rho^{\prime}} l_{2}-\frac{2 \sigma}{\Delta \rho^{\prime} g r},
$$

where $\Delta \rho^{\prime} \equiv \rho_{C}^{\prime}-\rho_{N}$. Note that if $\rho_{C}^{\prime}=\rho_{C}$, then the first term vanishes and the usual capillary-rise formula (1) is recovered. Using Eq. (5) and making the approximation $\Delta \rho^{\prime} \simeq \Delta \rho$, Eq. (7) becomes

$$
H \simeq-\frac{2 \delta l_{2}}{r}-\frac{2 \sigma}{\Delta \rho g r}
$$

This simple model, that the wetting layer response to a temperature perturbation results in a capillary-rise change given approximately by Eq. (8), can be compared with our experiments. We first note that several qualitative trends are consistent. A temperature burn (quench) will cause a negative (positive) $\delta$, and consequently Eq. (8) predicts that the meniscus will move in the receding (advancing) direction; this agrees with Figs. 11-14. Also consistent with these observations is the prediction of Eq. (8) that the response will be greater for larger temperature changes, larger $l_{2}$, and smaller $r$. In fact, the ratio of 1.6 \pm 0.3 observed in Fig. 13 for the response for two different values of $l_{2}$ is very close to the prediction of Eq. (8): $4.4 \mathrm{~cm} / 2.4 \mathrm{~cm}=1.8$. Remarkably; the observed response (about $1 \mathrm{~mm}$ ) is only one order of magnitude larger than the prediction of our simple model: for $\delta=100 \mathrm{~nm},{ }^{25,26} l_{2}=4.4 \mathrm{~cm}$, and $r=0.134 \mathrm{~mm}$, Eq. (8) gives a capillary rise change of $2 \delta l_{2} / r \simeq 0.07 \mathrm{~mm}$.

To test the model more thoroughly, it would be prefer- able to reequilibrate at the same temperature and apply temperature jumps of various sizes, rather than perform successive jumps. It may be necessary to refine the model by including the dependence of the wetting layer thickness, ${ }^{27}$ and its sensitivity to temperature perturbations, ${ }^{25}$ on height along the capillary tube.

\section{POSSIBLE EXPLANATIONS OF THE INVERTED MENISCUS}

We now present scenarios by which the wetting layer inside the capillary tube could invalidate the usual capillary rise formula (1) and produce an inverted meniscus. We first note that Ref. 2 previously found that a wetting layer can influence the capillary rise. There, the layer acted to reduce the capillary separation and caused a larger rise than would otherwise be expected. However, this effect changes the rise in the wrong direction to explain the inverted meniscus. We next note that since the inverted meniscus has been observed in three different binary liquid mixtures (one with wetting by the upper phase and a positive $T_{c}$ drift; one with wetting by the lower phase and an initially negative $T_{c}$ drift; and one with wetting by the lower phase, no $T_{c}$ drift, and an inverted coexistence curve), it is unlikely that temperature gradients, $T_{c}$ drifts, or exotic substrate-liquid interactions are responsible (nevertheless, these possibilities might be interesting to pursue). Rather, we speculate that a stationary inverted meniscus could occur sufficiently close to the critical temperature of any binary mixture. Three possible explanations will now be outlined, all inspired by our previous conclusion that a change in wetting layer thickness can affect the hydrostatic balance and perturb the capillary rise.

In the first scenario, the imperfect temperature control provided by the experimental thermostat produces an inverted meniscus through a pumping action. When the temperature wanders toward $T_{c}$ the meniscus recedes toward an inverted configuration, just as in the temperature jump experiments. Deficit wetting layer material is then partly replenished by flow along the wall of the capillary so that temperature fluctuations back away from $T_{c}$ do not draw as much material out of the interior of the capillary. A steady state may eventually be reached in which wetting layer material is continually pumped into, then slowly diffuses out of, the nonwetting phase inside the capillary tube, making it rich enough in wetting layer material to cause an inverted meniscus.

Another scenario in which imperfect temperature control may produce an inverted meniscus arises from a possible asymmetry between advancing and receding contact line motion (as seen near $T_{c}$ in Fig. 2). As the sample temperature fluctuates, the resulting change in wetting layer thickness gives rise to a randomly fluctuating hydrostatic force on the meniscus inside the capillary. If there is less friction when a contact line moves in the receding direction, the random force will produce a net displacement in the capillary rise in the proper direction for an inverted meniscus. Contrary to the first scenario, here the average densities of the liquid phases inside the capillary are equal to the average densities outside. 
We have thus imagined two scenarios in which random temperature perturbations are rectified by the system to produce a net displacement of the interface and, possibly, an inverted meniscus. The temperature oscillation experiment of Fig. 14 was conceived to test the hypothesis of rectification, but none was observed. This tends to rule out these two scenarios; however, our perturbation was at such low frequency that the wetting layer response was large and in phase with the temperature. Another experimental test would be to increase the amplitude of the driving temperature while keeping its frequency spectrum equal to that which normally occurs in the thermostat.

The third scenario does not depend on the rectification of temperature perturbations. Here we propose that the hydrostatic balance may be altered due to a nonequilibrium wetting layer thickness left after the contact line travels inward from one end. For the case of a receding meniscus, the flow could leave behind a wetting layer whose thickness is too great. ${ }^{28}$ It would subsequently thin down, changing the liquid density inside the capillary in the proper direction for an inverted meniscus. For the case of an advancing meniscus, a precursor film exists ahead of the traveling interface. ${ }^{7}$ In order to explain the inverted meniscus, its thickness would have to be greater than that of the equilibrium wetting layer.

A dimensional measure of interface motion of speed $V$ is the capillary number $\mathcal{N}_{\mathrm{Ca}}=\eta V / \sigma$. For the exponential relaxation of Eq. (3), it is given by

$$
\mathcal{N}_{\mathrm{Ca}}(\tau)=\frac{\left|H(\tau)-H_{\mathrm{eq}}\right| r}{4 H_{\mathrm{eq}} L} .
$$

Even though such flows have small Reynold's numbers, as discussed earlier, their capillary numbers can become large near $T_{c}$ as the surface tension vanishes. Equation (9) shows that very large $\mathcal{N}_{\mathrm{Ca}}$ (of order unity) are attained if the distance from equilibrium is comparable to $L$ and if the equilibrium rise value is less than the radius. For $\mathcal{N}_{\mathrm{Ca}}>10^{-3}$, viscous forces near the wall are already strong enough to change the shape of the interface, producing a dynamic contact angle which significantly differs from the static value. ${ }^{7,29,30}$ During our large- $\mathcal{N}_{\mathrm{Ca}}$, small- $\mathcal{N}_{\mathrm{Re}}$, flows, we noticed that the meniscus became very flat in the center of the capillary for both advancing and receding cases. When such a flow stops, it is conceivable that the wetting layer will have a thickness other than its equilibrium value. Note that this scenario would imply a history dependence which could explain the scatter found in Figs. 8 and 9.

Contrary to the rectification scenarios, here the inverted meniscus is a not steady-state phenomenon. It would persist up to a diffusion time for the length of the capillary tube: $L^{2} / D$ where $L=6 \mathrm{~cm}$ in our experiments and $D$ is the diffusion constant. Far from $T_{c}$, where $D$ is on the order of $10^{-5} \mathrm{~cm}^{2} / \mathrm{s}$, this time is already about $40 \mathrm{~d}$. Near $T_{c}$ this can become even longer due to critical slowing down. ${ }^{31}$ For carbon disulfide plus nitromethane at 30 $\mathrm{mK}$ below $T_{c}$, we have $D \simeq 2 \times 10^{-7} \mathrm{~cm}^{2} / \mathrm{s}$ and $L^{2} / D \simeq 2100 \mathrm{~d} .^{32}$ Our experiments were of insufficient duration to test this scenario.

Finally, it is worthwhile to emphasize that in the above scenarios, the capillary rise deviates from the usual capillary-rise formula, Eq. (1), in response to a change in hydrostatic conditions, not due to a change in contact angle. Therefore the standard methods of direct visual observation of the contact angle are, in principle, applicable even under conditions that an inverted meniscus is obtained.

\section{CONCLUSIONS}

Capillary-rise experiments for the purpose of determining contact angles should be performed with a given capillary only sufficiently far from $T_{c}$ that the rise is much greater than the radius. To study wetting phenomena closer to $T_{c}$, a smaller radius capillary tube must be used. In order to make acccurate measurements, it is important to determine the characteristic relaxation time and to check that the same capillary rise is obtained for advancing and receding conditions and for different positions of the capillary with respect to the bulk meniscus. Close enough to $T_{c}$, for a particular capillary, (i) these checks will fail, (ii) the relaxation time will grow long, (iii) the measurements will be sensitive to small drifts or perturbations in the temperature control, and (iv) an inverted meniscus may appear. The origin of (iii), and possibly (iv), is that a wetting layer thickness change can affect the hydrostatic force balance which determines the capillary rise.

\section{ACKNOWLEDGMENTS}

Throughout this work we have greatly benefited from the suggestions, encouragement, and interest of $M$. E. Fisher and B. Widom. We thank B. Delibero, C. Keller, M. Schlossman, and X.-1. Wu, for assistance, and E. Herbolzheimer, A. J. Liu, and D. Ripple for helpful conversations. This work was supported by the National Science Foundation through the Materials Science Center at Cornell University, and through Low Temperature Physics Grant No. DMR-8611350. The support of S.K.W. by the TRW Corporation is gratefully acknowledged.
*Formerly Kumudini Abeyaratne; present address: 26 Donny Ave., Hamilton, New Zealand.

${ }^{1}$ D. H. Pohl and W. I. Goldburg, Phys. Rev. Lett. 48, 1111 (1982).

${ }^{2}$ M. R. Moldover and R. W. Gammon, J. Chem. Phys. 80, 528 (1985).

${ }^{3}$ L. Sigl and W. Fenzl, Phys. Rev. Lett. 57, 2191 (1986).
${ }^{4}$ K. Abeysuriya, X.-1. Wu, and C. Franck, Phys. Rev. B 35, 6771 (1987).

${ }^{5}$ D. J. Durian and C. Franck, Phys. Rev. Lett. 59, 555 (1987); 59, 1492 (1987).

${ }^{6}$ D. J. Durian and C. Franck, Phys. Rev. B 36, 7307 (1987).

${ }^{7}$ P. G. de Gennes, Rev. Mod. Phys. 57, 827 (1985).

${ }^{8}$ D. E. Sullivan and M. M. Telo da Gama, in Fluid Interfacial 
Phenomena, edited by C. A. Croxton (Wiley, London, 1986), p. 72.

${ }^{9} \mathrm{~S}$. Dietrich, in Phase Transitions and Critical Phenomena, edited by C. Domb and J. L. Lebowitz (Academic, London, 1988), Vol. 12, p. 1.

${ }^{10}$ Articles by D. Beysens and by M. Schick in Liquids at Interfaces, 1988 Les Houches Lectures, edited by J. Charvolin and J. F. Joanny (North-Holland, Amsterdam, 1990).

${ }^{11} \mathrm{~J}$. S. Rowlinson and B. Widom, Molecular Theory of Capillarity (Clarendon, Oxford, 1982).

${ }^{12}$ A. W. Adamson, Physical Chemistry of Surfaces (Wiley, New York, 1982).

${ }^{13}$ K. Abeyaratne, M. S. thesis, Cornell University, 1986, pp. 66-68.

${ }^{14}$ Item numbers 22,704-8; 23,073-1; 24,284-5; 27,066-0; 27,073-3; and L390-0, Aldrich Chemical Company, Milwaukee, WI.

${ }^{15}$ Item numbers 21-164-2B, C, and E, Fisher Scientific Company, Pittsburg, PA.

${ }^{16}$ Aqueous solution of ethylenediamine tetra-acetic acid adjusted to $p \mathbf{H}=8.5$ by sodium hydroxide.

${ }^{17}$ E. S. R. Gopal, P. Chandra Sekhar, G. Ananthakrishna, R. Ramachandra, and S. V. Subramanyam, Proc. R. Soc. London Ser. A 350, 91 (1976).

${ }^{18}$ A. W. Loven and O. K. Rice, Trans. Faraday Soc. 59, 2723 (1963).

${ }^{19}$ Two groups have repeatedly observed wetting of glass substrates by the lutidine-rich phase: V. Gurfein, D. Beysens, and F. Perrot, Phys. Rev. A 40, 2543 (1989), on colloidal silica; and P. Wiltzius, S. B. Dierker, and B. S. Dennis, Phys. Rev. Lett. 62, 804 (1989), on Vycor over most of the concentration range. We have found that borosilicate glass capillaries altered by reaction with $1 \%$ heptyltrichlorosilane in dicyclohexyl were wetted by the lutidine-rich phase.

${ }^{20}$ M. E. Fisher and J.-H. Chen, J. Phys. (Paris) 46, 1645 (1985).

${ }^{21}$ No. KM-221, Wild Heerbrugg, Switzerland.

${ }^{22}$ An advancing meniscus occurs when the wetting phase displaces the nonwetting phase, and vice versa for a receding meniscus.

${ }^{23}$ E. W. Washburn, Phys. Rev. 17, 273 (1921).

${ }^{24}$ T. E. Mumley, C. J. Radke, and M. C. Williams, J. Colloid Interface Sci. 109, 398 (1986).

${ }^{25}$ D. J. Durian and C. Franck, Phys. Rev. A 40, 5220 (1989).

${ }^{26}$ X.-1. Wu, D. Ripple, and C. Franck, Phys. Rev. A 36, 3975 (1987).

${ }^{27}$ R. F. Kayser, J. W. Schmidt, and M. R. Moldover, Phys. Rev. Lett. 54, 707 (1985).

${ }^{28}$ M. O. Robbins and J. M. di Meglio (independent communications).

${ }^{29}$ E. B. Dussan V., Annu. Rev. Fluid Mech. 11, 371 (1979).

${ }^{30}$ M.-Y. Zhou and P. Sheng, Phys. Rev. Lett. 64, 882 (1990).

${ }^{31}$ P. C. Hohenberg and B. I. Halperin, Rev. Mod. Phys. 49, 435 (1977).

${ }^{32}$ Footnote 7 of Ref. 26. 


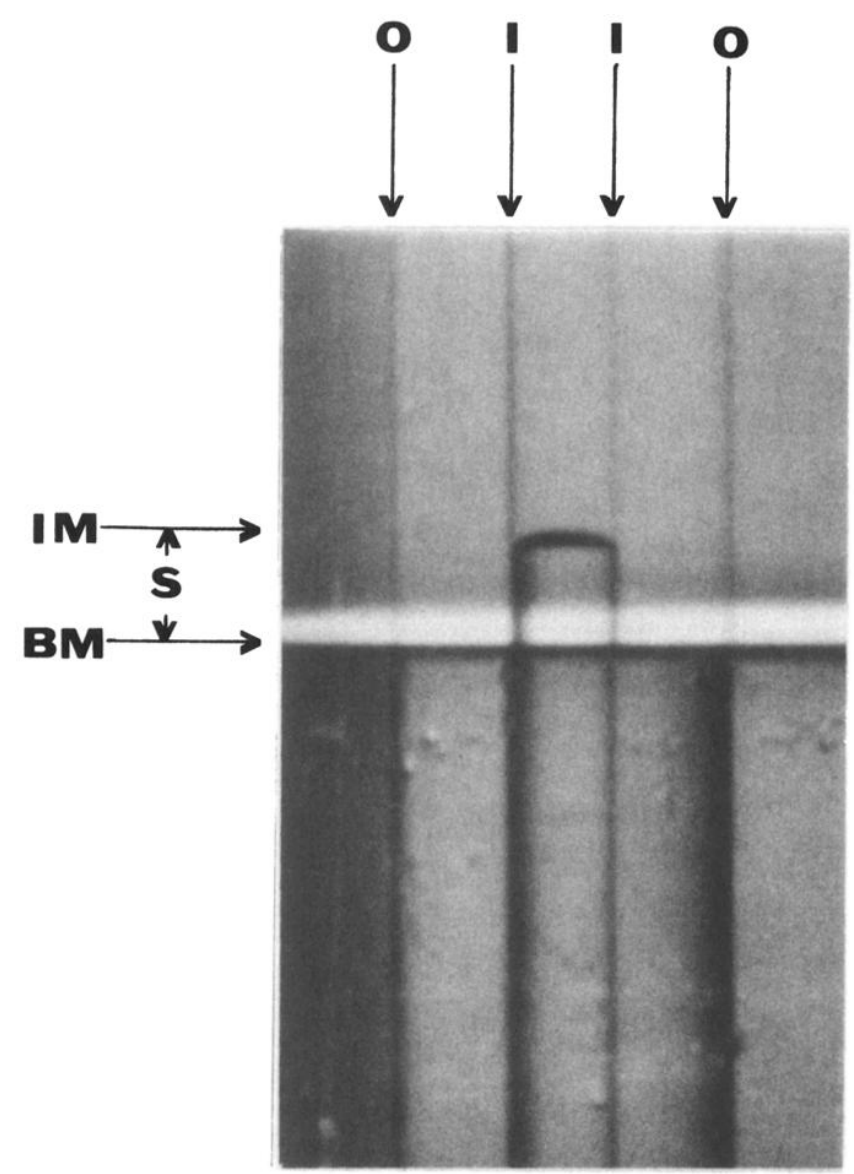

FIG. 5. Photograph of the inverted meniscus in a $0.188-\mathrm{mm}-$ inner-radius capillary at $35 \mathrm{mK}$ below the critical temperature of a carbon disulfide plus nitromethane mixture. $\mathrm{O}$ (I) indicates the outer (inner) wall of the capillary tube; BM and IM indicate the respective bulk and inverted meniscus positions; and $\mathrm{s}$ is the capillary rise. Note that $s$ is positive even though the meniscus curvature is clearly visible and implies wetting by the upper phase. 